\title{
Charged pions probing strong CP violation in chiral-imbalance medium?
}

\author{
Mamiya Kawaguchi $^{* 1}$, Masayasu Harada ${ }^{1}$, Shinya Matsuzaki ${ }^{1,2}$ and Ruiwen Ouyang ${ }^{3}$ \\ ${ }^{1}$ Department of Physics, Nagoya University, Nagoya 464-8602, Japan. \\ ${ }^{2}$ Institute for Advanced Research, Nagoya University, Nagoya 464-8602, Japan. \\ ${ }^{3}$ College of Physics, Jilin University, Changchun, 130012, China \\ E-mail: mkawaquchiehken.phys.nagova-u.ac.jp, \\ haradadhken.phys.naqova-u.ac.jp \\ synyadhken.phys.nagova-u.ac.ip \\ ruiwen.ouyangegmail.com
}

We propose a novel probe for the strong $\mathrm{CP}$ violation: the charged-pion form factor could pick up the remnant of the $\mathrm{CP}$ violation in the chiral-imbalance medium, which can be produced in heavy ion collisions. Such a charged-pion profile could be observed by the parity violation of direct photon detection measurements at heavy ion collision experiments.

The 3rd International Symposium on "Quest for the Origin of Particles and the Universe" 5-7 January 2017

Nagoya University, Japan

\footnotetext{
* Speaker.
} 


\section{Introduction}

QCD has topologically non-trivial gauge field configurations characterized by the winding number. The configurations break the $\mathrm{CP}$ symmetry (strong $\mathrm{CP}$ violation). Placing the QCD in hot and/or dense environments would be an intriguing proposal to access the strong $\mathrm{CP}$ detection, because of the presence of the thermally excited-topologically nontrivial gauge configuration (socalled the sphaleron [U, []]), which would make the lifetime of the CP-violating local domain long

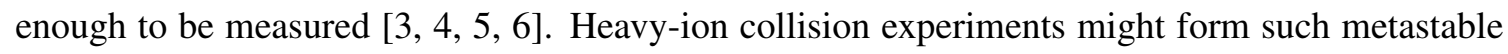
domains where $\mathrm{P}$ - and CP-symmetries are locally violated, as has so far been addressed in the

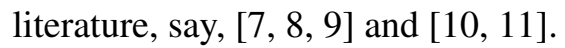

In this presentation, we propose a novel possibility to observe the strong $\mathrm{CP}$ violation in the chiral-imbalance medium. It is the charged-pion profile coupled to the electromagnetic field in the chiral-imbalance medium, which can be realized in heavy ion collision experiments. Such a charged-pion profile could be seen through the parity violation of direct photon detection measurements with the charged pion at heavy ion collision experiments. This work is based on the paper [12].

\section{Short review of the chiral-imbalance medium with chiral chemical potential}

In this section, we briefly review the chiral imbalance medium characterized in the presence of the chiral chemical potential $\mu_{5}$. It is associated to the appearance of local fluctuations of the topological charge, and we can then interpret its fluctuation as the difference between the number of right-handed and left-handed quarks.

In heavy ion collision experiments, the fireball is created at the time scale $\tau_{\text {fireball }}$ of $\mathscr{O}(1-10)$ $\mathrm{fm}$ after the collision [[3]]. In the fireball bulk, it is supposed that the QCD $\theta$ vacuum has the position-space time dependence, which can be rephrased by the position-space time dependence

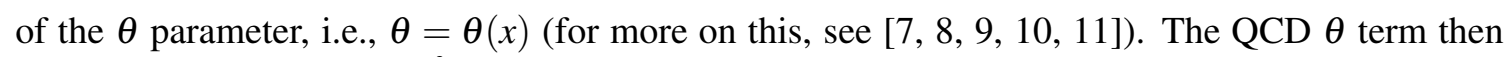
looks like $\int_{\text {bulk }} d^{4} x \theta(x) \cdot \frac{g_{s}^{2}}{32 \pi^{2}} \varepsilon^{\mu \nu \rho \sigma} \operatorname{tr}\left[G_{\mu \nu} G_{\rho \sigma}\right]$, where the symbol "bulk" denotes the volume of the fireball bulk and $G_{\mu \nu}$ is the gluon-field strength, $G_{\mu \nu}=\partial_{\mu} G_{v}-\partial_{\nu} G_{\mu}-i g_{s}\left[G_{\mu}, G_{v}\right]$ with the QCD gauge coupling $g_{s}$. One should recall that the $\theta$ term is related to the axial-anomaly form

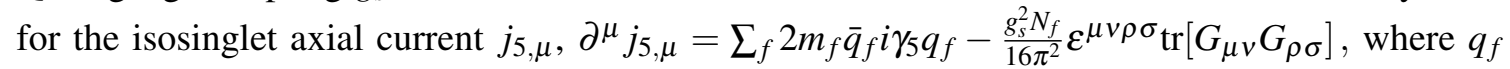
denotes the quark field, $m_{f}$ the quark mass, $N_{f}$ the number of the quark flavors. Using this anomaly equation, one finds that the QCD $\theta$ term goes like

$$
\int_{\text {bulk }} d^{4} x\left(\frac{\partial_{\mu} \theta(x)}{2 N_{f}}\right) \cdot j_{5}^{\mu}(x)+\cdots
$$

(" $\ldots$ " include the form $\theta \cdot m_{f}$ times pseudoscalar density and the surface terms) Looking at Eq.(D... one may identify the quantity in the bracket as the source field $\left(\mathscr{A}_{\mu}^{0}(x)\right)$ for the isosinglet axialvector current $j_{5}^{\mu}(x)[\mathbb{\square}, \mathbb{Q}, \mathbb{Q}, \mathbb{\square}, \mathbb{Z}, \mathbb{W}]:\left(\frac{\partial_{\mu} \theta(x)}{2 N_{f}}\right)=\mathscr{A}_{\mu}^{0}(x)$. To relate to the chiral chemical potential we shall assume that the $\theta(x)$ in the fireball bulk fluctuates only in time with the constant velocity,

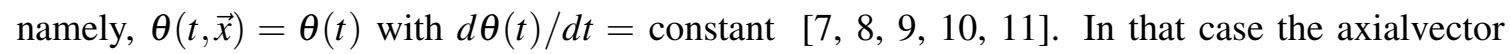
source $\mathscr{A}_{\mu}^{0}$ acts as if as a chemical potential, which is called the chiral chemical potential $\mu_{5}$ [Q]]: $\mathscr{A}_{\mu}^{0}=\left(\mu_{5}, \overrightarrow{0}\right)^{T}$. The hot QCD bulk, characterized by this $\mu_{5}$, would be like a medium, that is what we call the chiral-imbalance medium. 


\section{Charged pion form factor with $\mu_{5}$}

In the presence of the chiral chemical potential $\mu_{5}$, the parity symmetry as well as the Lorentz symmetry are broken to the three-dimensional $S O(3)$ symmetry. In that case, the general expression for the form factors of the charged pion coupled to the background photon field $A^{\mu}$ with the transfer momentum $q$ is written as

$$
\begin{aligned}
\left\langle\pi^{+}\left(p^{\prime}=q+p\right)\left|\mathscr{J}^{\mu}(0)\right| \pi^{+}(p)\right\rangle= & e F_{1} \cdot\left(p^{0}+p^{\prime 0}\right) \delta_{0}^{\mu}+e F_{2} \cdot\left(p^{i}+p^{i}\right) \delta_{i}^{\mu} \\
& +e F_{3} \cdot\left(q^{i}\right) \delta_{i}^{\mu}+i e F_{4} \cdot \varepsilon^{0 \mu v \rho} p_{v} p_{\rho}^{\prime},
\end{aligned}
$$

where $\mathscr{J}^{\mu}$ is the electromagnetic current including nonminimal forms, $e$ the electromagnetic coupling and $F_{i=1,2,3,4}=F_{i=1,2,3,4}\left(q^{0}, p^{0},|\vec{q}|,|\vec{p}|\right)$ denote the form factors. In terms of the effective action, the first term and second term correspond to the coefficients of the $\pi^{+}-\pi^{-}-\gamma$ interactions like $A^{0} j^{0}$ and $\vec{A} \cdot \vec{j}$, respectively, where $j^{\mu}$ is the pion electromagnetic current of the minimal coupling form: $j_{\mu}=i e\left(\partial_{\mu} \pi^{+} \cdot \pi^{-}-\partial_{\mu} \pi^{-} \cdot \pi^{+}\right)$. By imposing the Ward-Takahashi identity for the electromagnetic gauge invariance, the third term can be written as linear combination of the other form factors. The fourth term $F_{4}$ is the one that will be induced in the presence of $\mu_{5}$ in the chiral-imbalance medium.

To see the explicit form of the $F_{4}$-form factor in the chiral-imbalance medium,we employ the chiral Lagrangian based on the coset space $G / H=\left[U(2)_{L} \times U(2)_{R}\right] / U(2)_{V}$ and the Wess-ZuminoWitten (WZW) term [14]]:

$$
S=\int d^{4} x \frac{f_{\pi}^{2}}{4} \operatorname{tr}\left[D_{\mu} U^{\dagger} D^{\mu} U\right]+S_{\mathrm{WZW}}
$$

where $f_{\pi}$ is the pion decay constant, $U$ denotes the chiral field parameterizing the pion fields as $U=\exp \left(\sum_{a=1}^{3} 2 i \pi^{a}\left(\sigma^{a} / 2\right) / f_{\pi}\right)$ with $\sigma^{a}$ being the Pauli matrices. The covariant derivative acting on $U$ is defined as $D_{\mu} U=\partial_{\mu} U-i \mathscr{L}_{\mu} U+i U \mathscr{R}_{\mu}$ with the external gauge fields $\mathscr{L}_{\mu}$ and $\mathscr{R}_{\mu}$. The photon field and the chiral chemical potential $\mu_{5}$ are then introduced as a part of the external vector and axialvector gauge fields $\left(\mathscr{V}_{\mu}, \mathscr{A}_{\mu}\right)$ as $\mathscr{V}_{\mu}=\frac{1}{2}\left(\mathscr{R}_{\mu}+\mathscr{L}_{\mu}\right)=e Q_{\mathrm{em}} A_{\mu}, \mathscr{A}_{\mu}=\frac{1}{2}\left(\mathscr{R}_{\mu}-\mathscr{L}_{\mu}\right)=$ $\mu_{5} \delta_{\mu 0} \cdot 1_{2 \times 2}$, where the electromagnetic charge matrix is defined as $Q_{\mathrm{em}}=\sigma_{3} / 2+1_{2 \times 2} / 6$. In $\mathrm{Eq}$.(B.2]), the second term is the WZW term including the following interactions between the pion and external gauge fields:

$$
\begin{aligned}
S_{\mathrm{WZW}}= & i \frac{N_{c}}{48 \pi^{2}} \int d^{4} x \varepsilon^{\mu v \rho \sigma_{\operatorname{tr}}}\left[\left\{\partial_{\mu} \mathscr{L}_{v}, \mathscr{L}_{\rho}\right\}\left(\partial_{\sigma} U\right) U^{\dagger}+\left\{\partial_{\mu} \mathscr{R}_{v}, \mathscr{R}_{\rho}\right\} U^{\dagger} \partial_{\sigma} U-\partial_{\mu} \mathscr{L}_{v} \partial_{\rho} U \mathscr{R}_{\sigma} U^{\dagger}\right. \\
& +\partial_{\mu} \mathscr{R}_{v} \partial_{\rho} U^{\dagger} \mathscr{L}_{\sigma} U-\mathscr{R}_{\mu} U^{\dagger} \mathscr{L}_{v}\left(\partial_{\rho} U\right) U^{\dagger} \partial_{\sigma} U+U^{\dagger} \mathscr{L}_{\mu} U \mathscr{R}_{v} U^{\dagger}\left(\partial_{\rho} U\right) U^{\dagger}\left(\partial_{\sigma} U\right) \\
& \left.-\frac{1}{2} \mathscr{L}_{\mu}\left(\partial_{v} U\right) U^{\dagger} \mathscr{L}_{\rho}\left(\partial_{\sigma} U\right) U^{\dagger}+\frac{1}{2} \mathscr{R}_{\mu}\left(\partial_{v} U\right) U^{\dagger} \mathscr{R}_{\rho}\left(\partial_{\sigma} U\right) U^{\dagger}\right]+\cdots,
\end{aligned}
$$

with the number of colors $N_{c}=3$. We extract the interactions of the charged pion coupled to the electromagnetic field in the chiral-imbalance medium to get

$$
D_{\mu} \pi^{+} D^{\mu} \pi^{-}+\frac{i e \mu_{5} N_{c}}{6 \pi^{2} f_{\pi}^{2}} \varepsilon^{0 v \rho \sigma} \partial_{v} \pi^{+} \partial_{\rho} \pi^{-} A_{\sigma},
$$

where $D_{\mu} \pi^{ \pm}=\left(\partial_{\mu} \mp i e A_{\mu}\right) \pi^{ \pm}$. Obviously, the second term breaks the parity. From Eq.(B. (B) we read off the electromagnetic form factors corresponding to the general expressions in Eq.(B.].

$$
F_{1}=F_{2}=1, F_{3}=0, F_{4}=\frac{\mu_{5} N_{c}}{6 \pi^{2} f_{\pi}^{2}} .
$$


The result on $F_{1}=1$ in Eq.(B.5) just tells us that the pion electromagnetic charge is correctly normalized, which just reflects the electromagnetic gauge invariance, while the normalization for $F_{2}=1$ has happened by accident. It is interesting to note that, although the pion is the spin 0 particle, the nonzero $F_{4}$ term looks like the anomalous magnetic moment of the charged pion.

\section{Phenomenological consequence}

The phenomenological consequence of $F_{4}$ could be observed by photon helicities along with the charged pions with the opening angle dependence. This would be measured from processes such as $\pi^{ \pm} \gamma \rightarrow \pi^{ \pm} \gamma$, where the charged pion and photon in the initial state come from the chiralimbalance medium. To observe the presence of the parity breaking piece, we should define the asymmetry value $(\mathscr{A})$ which is proportional to the difference of the number densities $\mathscr{N}_{ \pm}=$ $d N\left(\pi^{+} \gamma_{ \pm} \rightarrow \pi^{+} \gamma_{ \pm}\right) / d \Omega$, where the subscript " \pm " is photon helicities $\left(s_{z}=+,-\right)$ and $\Omega$ is the body angle for the $\pi^{+}$and $\gamma_{ \pm}$-final sates. The asymmetry $\mathscr{A}$ for the chiral chemical potential $\mu_{5}$ is roughly estimated as

$$
\mathscr{A}_{\mu_{5}} \equiv\left|\frac{\mathscr{N}_{+}-\mathscr{N}_{-}}{\mathscr{N}_{+}+\mathscr{N}_{-}}\right|=\mathscr{O}\left(\frac{\mu_{5}\left|p_{\pi}\right| N_{c}}{6 \pi^{2} f_{\pi}^{2}}\right)=\mathscr{O}\left(10^{-1}\right) \times\left(\frac{\mu_{5}}{200 \mathrm{MeV}}\right),
$$

where $\left|p_{\pi}\right|$, the size of the pion momentum was taken to be $\mathscr{O}(100 \mathrm{MeV})$. Expected backgrounds for this parity violating event would be dominated by the electroweak interaction process. At the leading order of electroweak perturbation, this roughly yields $\mathscr{A}_{\mathrm{EW}}^{\mathrm{Bkgd}}=\mathscr{O}\left(N_{c} /(4 \pi)^{2}\left(G_{F} f_{\pi}\right)^{2}\right)=$ $\mathscr{O}\left(10^{-9}\right)$, where $G_{F}$ is the Fermi constant $\simeq(290 \mathrm{GeV})^{-2}$. If the size of the scale $\mu_{5}$ would be on the order of $\Lambda_{\mathrm{QCD}}$, the parity breaking effect in Eq.(14) would give the distinct signal.

\section{Summary}

We have propounded a new probe for measuring the strong CP violation. It is the chargedpion form factor that could pick up the remnant of the $\mathrm{CP}$ violation in the chiral-imbalance medium, which can be produced in heavy ion collisions. Our proposal presented here could be tested, for instance, through the parity violation of direct photon detection measurements along with charged pions in heavy ion collision experiments with high statistics.

\section{Acknowledgments}

This work was supported in part by the JSPS Grant-in-Aid for Young Scientists (B) No. 15K17645 (S.M.), and the JSPS Grant-in-Aid for Scientific Research (C) No. 16K05345 (M.H.). R.O. was partially supported by the TAQ honor program in physics from the Office of Undergraduate Education and College of Physics in Jilin University.

\section{References}

[1] N. S. Manton, Phys. Rev. D 28, 2019 (1983). doi:10.1103/PhysRevD.28.2019.

[2] F. R. Klinkhamer and N. S. Manton, Phys. Rev. D 30, 2212 (1984). doi:10.1103/PhysRevD.30.2212. 
[3] L. D. McLerran, E. Mottola and M. E. Shaposhnikov, Phys. Rev. D 43, 2027 (1991). doi:10.1103/PhysRevD.43.2027.

[4] G. D. Moore, Phys. Lett. B 412, 359 (1997) doi:10.1016/S0370-2693(97)01046-0.

[5] G. D. Moore and K. Rummukainen, Phys. Rev. D 61, 105008 (2000) doi:10.1103/PhysRevD.61.105008.

[6] D. Bodeker, G. D. Moore and K. Rummukainen, Phys. Rev. D 61, 056003 (2000) doi:10.1103/PhysRevD.61.056003.

[7] D. Kharzeev and A. Zhitnitsky, Nucl. Phys. A 797, 67 (2007) doi:10.1016/j.nuclphysa.2007.10.001.

[8] D. E. Kharzeev, L. D. McLerran and H. J. Warringa, Nucl. Phys. A 803, 227 (2008) doi:10.1016/j.nuclphysa.2008.02.298.

[9] K. Fukushima, D. E. Kharzeev and H. J. Warringa, Phys. Rev. D 78, 074033 (2008) doi:10.1103/PhysRevD.78.074033.

[10] A. A. Andrianov, V. A. Andrianov, D. Espriu and X. Planells, Phys. Lett. B 710, 230 (2012) doi:10.1016/j.physletb.2012.02.072.

[11] A. A. Andrianov, D. Espriu and X. Planells, Eur. Phys. J. C 73, no. 1, 2294 (2013) doi:10.1140/epjc/s10052-013-2294-0.

[12] M. Kawaguchi, M. Harada, S. Matsuzaki and R. Ouyang, arXiv:1612.00616 [nucl-th].

[13] R. Derradi de Souza, T. Koide and T. Kodama, Prog. Part. Nucl. Phys. 86, 35 (2016) doi:10.1016/j.ppnp.2015.09.002 [arXiv:1506.03863 [nucl-th]].

[14] J. Wess and B. Zumino, Phys. Lett. 37B, 95 (1971). doi:10.1016/0370-2693(71)90582-X; E. Witten, Nucl. Phys. B 223, 422 (1983). doi:10.1016/0550-3213(83)90063-9. 